\title{
Quasi-elastic peak lineshapes in adsorbate diffusion on nearly flat surfaces at low coverages: the motional narrowing effect in Xe on $\mathrm{Pt}(111)$
}

\author{
R Martínez-Casado ${ }^{1,2}$, J L Vega ${ }^{2 *}$ A S Sanz ${ }^{2}$ and S Miret-Artés ${ }^{2}$ \\ ${ }^{1}$ Ruhr-Universität Bochum, Lehrstuhl für Physikalische Chemie I \\ D-44801 Bochum, Germany \\ ${ }^{2}$ Instituto de Matemáticas y Física Fundamental \\ Consejo Superior de Investigaciones Científicas \\ Serrano 123, 28006 Madrid, Spain.
}

\begin{abstract}
Quasi-elastic helium atom scattering measurements have provided clear evidence for a two-dimensional free gas of Xe atoms on $\mathrm{Pt}(111)$ at low coverages. Increasing the friction due to the surface, a gradual change of the shape of the quasi-elastic peak is predicted and analyzed for this system in terms of the so-called motional narrowing effect. The type of analysis presented here for the quasi-elastic peak should be prior to any deconvolution procedure carried out in order to better extract information from the process, e.g. diffusion coefficients and jump distributions. Moreover, this analysis also provides conditions for the free gas regime different than those reported earlier.
\end{abstract}

\section{Introduction}

One of the main observables in adsorbate diffusion on metal surfaces is the full width at half maximum (FWHM) of the quasi-elastic (Q-)peak as a function of the parallel momentum transfer, $\Delta \mathbf{K}=\mathbf{K}_{f}-\mathbf{K}_{i}$. Different magnitudes of interest can be easily extracted from it, e.g. diffusion coefficients and jump distributions. In the 1950s van Hove [1] and Vineyard [2] studied the broadening/narrowing of the Q-peak for several simple models (ranging

${ }^{*}$ Present address: Biosystems Group, School of Computing, University of Leeds, Leeds, LS2 9JT United Kingdom. 
from the free particle to a lattice atom and the liquid phase) in order to characterize the nature of the phase under study in terms of this property (if possible). However, no attention has been usually paid to the actual lineshape of the Q-peak. Is the shape of the Q-peak preserved when the surface friction and the parallel momentum transfer of the particles probing the adsorbates change? This is a critical question; a precise knowledge of the natural lineshape is crucial in the experimental deconvolution procedure for a better description of the diffusion mechanisms underlying the adsorbate dynamics. Nowadays, the wealth of experimental data available in surface science (obtained either by standard time-of-flight techniques [3, 4] or by spin-echo measurements [5]) allows us to tackle this question successfully, as we show in this work. In particular, quasi-elastic helium atom scattering (QHAS) has provided [4 the first experimental evidence for a fully mobile two-dimensional gas of $\mathrm{Xe}$ atoms on $\mathrm{Pt}(111)$ at low coverage $(\theta=0.017)$, low incident helium atom energy $\left(E_{i}=10.15 \mathrm{meV}\right)$ and a surface temperature $T_{s}=105 \mathrm{~K}$.

One of the theoretical approaches used to interpret the experimental results from QHAS measurements is the classical Langevin formalism [6, 7], sometimes combined with standard molecular dynamics techniques [4]. Here we are going to use such an approach in order to analyze the Xe$\mathrm{Pt}(111)$ system at the experimental conditions considered by Ellis et al. [4, analyzing the Q-peak lineshape in the light of the so-called motional narrowing effect [6, 8, 9]. As will be seen, this type of analysis should be prior to any deconvolution procedure aimed to better extract information from the process, such as diffusion coefficients or jump distributions.

The organization of this work is as follows. In order to be self-contained, in section 2 we present the theoretical approach that we have followed to obtain the analytical formulae that will allow us to interpret our numerical results; the numerical methodology is also presented in this section. The results derived from our approach are shown and discussed in section 3 , Finally, the main conclusions are summarized in section 4 .

\section{Theoretical approach}

From QHAS experiments one obtains the differential reflection coefficient which, in analogy to liquids [1, 10], can be expressed as

$$
\frac{d^{2} \mathcal{R}(\Delta \mathbf{K}, \omega)}{d \Omega d \omega}=n_{d} \mathcal{F} S(\Delta \mathbf{K}, \omega),
$$


and gives the probability that the He atoms scattered from the diffusing collective reach a certain solid angle $\Omega$ with an energy exchange $\hbar \omega=E_{f}-E_{i}$ and parallel (to the surface) momentum transfer $\Delta \mathbf{K}=\mathbf{K}_{f}-\mathbf{K}_{i}$. In the right hand side (r.h.s.) of equation (11), $n_{d}$ is the adparticle concentration; $\mathcal{F}$ is the atomic form factor, which depends on the interaction potential between the probe atoms and the adparticles; and $S(\Delta \mathbf{K}, \omega)$ is the so-called dynamic structure factor or scattering law, which is the observable magnitude in this type of experiments and provides complete information about the dynamics and structure of the ensemble of adparticles, and therefore also about the surface diffusion process. For example, information about long distance correlations is obtained from $S(\Delta \mathbf{K}, \omega)$ when considering small values of $\Delta \mathbf{K}$, while long timescale correlation information is available at small values of $\hbar \omega$.

The starting point of our approach consists of expressing the dynamic structure factor as [1, 6, 10]

$$
S(\Delta \mathbf{K}, \omega)=\int e^{-i \omega t} I(\Delta \mathbf{K}, t) d t
$$

where

$$
I(\Delta \mathbf{K}, t) \equiv\left\langle e^{-i \Delta \mathbf{K} \cdot[\mathbf{R}(t)-\mathbf{R}(0)]}\right\rangle=\left\langle e^{-i \Delta K \int_{0}^{t} v_{\Delta \mathbf{K}}\left(t^{\prime}\right) d t^{\prime}}\right\rangle
$$

is the intermediate scattering function, where the brackets denote (ensemble) averaging over the adsorbates' trajectories, $\mathbf{R}(t)$. In (3), $v_{\Delta \mathbf{K}}$ is the adparticle velocity projected onto the $\Delta \mathbf{K}$-direction (note that $\Delta K \equiv\|\Delta \mathbf{K}\|$ is the length of $\Delta \mathbf{K})$.

Both the dynamic structure factor and the intermediate scattering function can be readily obtained from Langevin numerical simulations as follows. Within a low coverage regime (as happens in Ellis et al. experiment [4], where $\theta=0.017$ ), adsorbate-adsorbate interactions can be neglected, and diffusion is fully characterized by only studying the dynamics of an isolated adsorbate on a metal surface. This is the so-called single adsorbate approximation. The adsorbate-substrate interaction includes the effects due to the surface corrugation as well as those arising from the surface thermal vibrational modes. Considering a temperature dependent expansion, both effects can be separated. Thus, one the one hand, there is an adiabatic, periodic adsorbate-substrate interaction potential $V$ which is temperature independent, namely the zero-temperature potential (i.e. $T_{s}=0 \mathrm{~K}$ ). On the other hand, there is a coupling term accounting for the vibrational effects induced by the temperature on the (surface) lattice atoms that act on the adsorbate, which can be substituted by a stochastic noise source [11]. This 
allows to use a Langevin approach [3], where the force acting on the adsorbates is given by two contributions: (1) a deterministic force $F=-\nabla V$, and (2) a stochastic force $G(t)$. As in standard Langevin molecular dynamics simulations [3, 4], the stochastic force has features of a Gaussian white noise (the diffusion process is considered as a Brownian-like motion [11]), i.e.

$$
\begin{aligned}
\langle G(t)\rangle & =0, \\
\left\langle G\left(t_{1}\right) G\left(t_{2}\right)\right\rangle & =2 m \gamma k_{B} T_{s} \delta\left(t_{2}-t_{1}\right),
\end{aligned}
$$

where $m$ is the adsorbate mass and $\gamma$ is the adsorbate-substrate coupling strength or friction coefficient.

Taking into account the previous discussion, the motion of an isolated adsorbate under the action of a bath consisting of the temperature-dependent surface vibrations can be characterized by the standard Langevin equation

$$
m \ddot{\mathbf{R}}(t)=-m \gamma \dot{\mathbf{R}}(t)+F(\mathbf{R}(t))+\mathbf{G}(t) .
$$

Here, $\mathbf{G}(t)$ is the two-dimensional (Gaussian white noise) stochastic force, whose components satisfy the conditions given by equations (4) and (5) moreover, $\left\langle G_{x}\left(t_{1}\right) G_{y}\left(t_{2}\right)\right\rangle=0$. Note that this equation of motion is based on the Markovian hypothesis: the correlations of the surface fluctuating force decay very rapidly [see equation (5)]. On the other hand, also notice that $\gamma$ gives rise to a characteristic timescale, the correlation time $\tau=1 / \gamma$, which is related to the mean free path that an adsorbate can travel without feeling much friction.

In order to interpret the Langevin numerical simulations, we can express the intermediate scattering function, given by r.h.s. of the second equality of equation (3), as a second order cumulant expansion in $\Delta K$,

$$
I(\Delta \mathbf{K}, t) \approx e^{-\Delta K^{2} \int_{0}^{t}\left(t-t^{\prime}\right) \mathcal{C}_{\Delta \mathbf{K}}\left(t^{\prime}\right) d t^{\prime}},
$$

where $\mathcal{C}_{\Delta \mathbf{K}}(t) \equiv\left\langle v_{\Delta \mathbf{K}}(0) v_{\Delta \mathbf{K}}(t)\right\rangle$ is the autocorrelation function of the velocity projected onto the direction of the parallel momentum transfer. This is the so-called Gaussian approximation [12, which is exact when the velocity correlations at more than two different times are negligible. Despite its limitations, it provides much insight into the dynamical process by allowing an almost analytical treatment of the problem. Note that it allows to replace the average acting over the exponential function by an average acting over its argument, thus simplifying the analytical derivation in some simple but relevant cases.

For an almost flat surface $(V \approx 0)$, any direction is equivalent and therefore the dimensionality of the numerical Langevin simulation reduces to one. 
The corresponding numerical velocity autocorrelation function then follows the exponential behavior

$$
\mathcal{C}(t)=\left\langle v_{0}^{2}\right\rangle e^{-\gamma t}
$$

where $\left\langle v_{0}^{2}\right\rangle=k_{B} T_{s} / m$ (the square root of this magnitude gives the thermal velocity in one dimension). Introducing (8) into equation (7), we obtain [9]

$$
I(\Delta \mathbf{K}, t)=\exp \left[-\chi^{2}\left(e^{-\gamma t}+\gamma t-1\right)\right],
$$

where $\chi$ is the shape parameter, defined as

$$
\chi \equiv \sqrt{\left\langle v_{0}^{2}\right\rangle} \Delta K / \gamma=\bar{l} \Delta K .
$$

From this relation the mean free path results $\bar{l} \equiv \tau \sqrt{\left\langle v^{2}\right\rangle}$, and the diffusion coefficient is $D \equiv \tau\left\langle v^{2}\right\rangle$, which related to the friction (Einstein's relation). It can be easily shown [6] that the dynamic structure factor derived from the Fourier transform of equation (9) has a different shape depending on the value of $\chi$. This can be seen from the exact (analytical) Fourier transform of equation (9), which renders the following functional form for the dynamic structure factor ruling the shape of the Q-peak

$$
\begin{aligned}
S(\Delta \mathbf{K}, \omega) & =\frac{\tau e^{\chi^{2}}}{\pi} \chi^{-2 \chi^{2}} \operatorname{Re}\left\{\chi^{-2 i \omega \tau}\left[\tilde{\Gamma}\left(\chi^{2}+i \omega \tau\right)-\tilde{\Gamma}\left(\chi^{2}+i \omega \tau, \chi^{2}\right)\right]\right\} \\
& =\frac{e^{\chi^{2}}}{2 \pi} \sum_{n=0}^{\infty} \frac{(-1)^{n} \chi^{2 n}}{n !} \frac{2\left(\chi^{2}+n\right) / \tau}{\omega^{2}+\left[\left(\chi^{2}+n\right) / \tau\right]^{2}}
\end{aligned}
$$

expressed in terms of the Gamma and incomplete Gamma functions (denoted by $\tilde{\Gamma}$ in the r.h.s. of the first line of equation (11) to avoid any confusion with the FWHM, $\Gamma$ ), respectively. According to this expression, as $\chi$ decreases (or, equivalently, as $\Delta K$ decreases, for $T_{s}$ and $\gamma$ fixed) the shape of the dynamic structure factor goes from a purely Gaussian function to a Lorentzian one, and its width gets narrower and narrower (see appendix). This is the so-called motional narrowing effect, well-known in the theory of nuclear magnetic resonance lineshapes [6, 8, 9]. The shape parameter goes from zero to infinity. At high values of $\chi$, the Q-peak approaches a Gaussian shape, which will be well reproduced only by few terms ( $n$ not too small) in the sum given by equation (11). For values of $\chi \ll 1$, the Q-peak approaches a Lorentzian shape, with the term corresponding to $n=0$ usually being the dominant one in equation (11). This variation between a Gaussian and a Lorentzian shape is in clear correspondence with having either a ballistic 


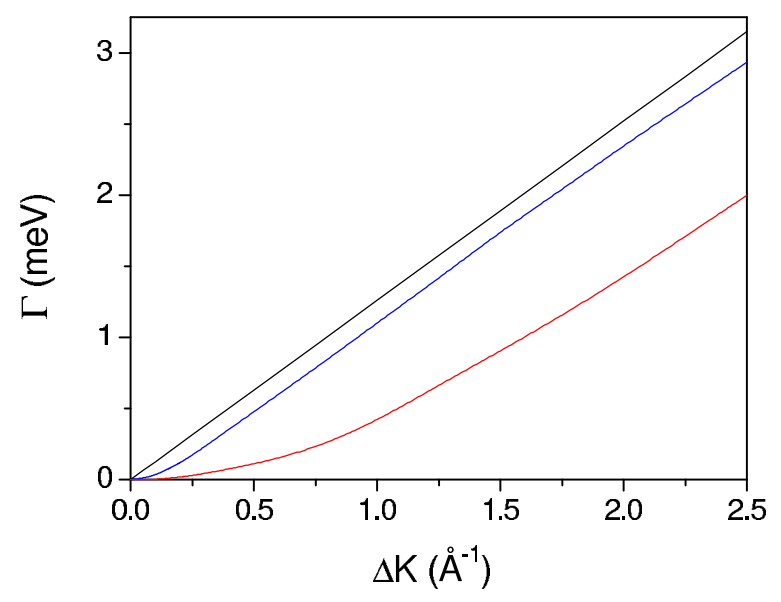

Figure 1: Full width at half maximum, $\Gamma$, of the quasi-elastic peak as a function of the parallel momentum transfer, $\Delta K$, along the direction (100) of the $\mathrm{Pt}(111)$ surface for three different values of the friction coefficient: $\gamma=0$ (black/thick black line), $\gamma=0.25 \mathrm{ps}^{-1}$ (blue/thin black line), and $\gamma=2.0 \mathrm{ps}^{-1}$ (red/thin grey line).

(free particle) or a diffusive regime, respectively. Thus, a simple manner of expressing the FWHM of the Q-peak, $\Gamma$, in terms of $\chi$ is

$$
\Gamma=2 \mu \gamma \chi^{2}+2 \sqrt{2 \ln 2}(1-\mu) \gamma \chi,
$$

with $\mu$ a free parameter. For $\mu=0$, one obtains the FWHM of the Gaussian lineshape, while the width corresponding to the Lorentzian lineshape is obtained for $\mu=1$ and when only the $n=0$ contribution of the infinite sum in equation (11) is considered (see appendix). That is, as the importance of the diffusive regime increases, one passes from a linear dependence on $\chi$ to a quadratic one. The same behavior is observed in $\Gamma$ when it is written as a function of $\Delta K$ because of the linear relationship between this magnitude and $\chi$ (see equation (10)).

\section{Results and discussion}

In figure 1, $\Gamma$ is plotted as a function of the parallel momentum transfer $\Delta K$ along the direction (100) for the Xe-Pt(111) system at $T_{s}=105 \mathrm{~K}$ and three different values of the friction coefficient $\left(0,0.25\right.$, and $\left.2 \mathrm{ps}^{-1}\right)$, assuming the corrugation of the $\mathrm{Pt}(111)$ surface negligible. These results 


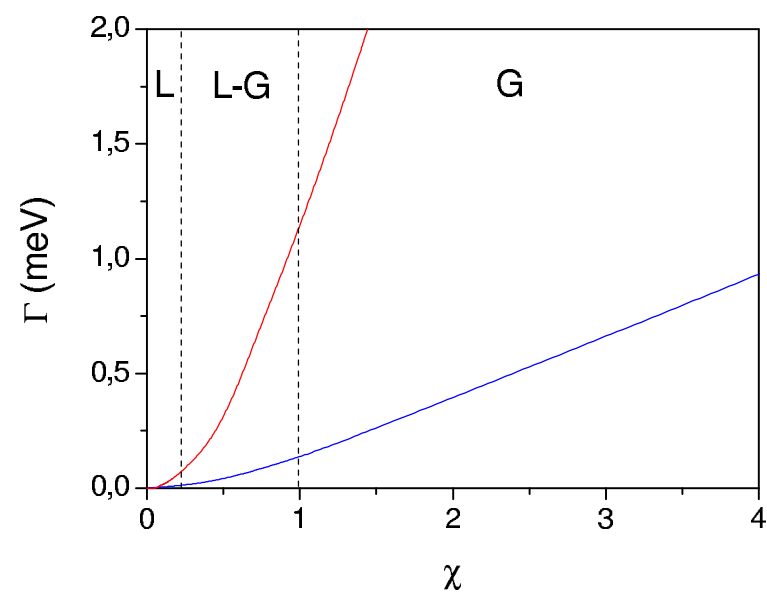

Figure 2: Full width at half maximum, $\Gamma$, of the quasi-elastic peak as a function of the shape parameter for two different values of the friction coefficient: $\gamma=0.25 \mathrm{ps}^{-1}$ (blue/thin black line) and $\gamma=2.0 \mathrm{ps}^{-1}$ (red/thin grey line). Vertical lines give the borders where a Lorentzian (L), a mixed Lorentzian-Gaussian (L-G), or a Gaussian (G) shape are observed.

are in excellent agreement with those obtained by Ellis et al. [4] by means of Langevin molecular dynamics simulations, i.e. considering interacting adsorbates, though the interaction among them is relatively weak because the coverage used in the experiment was relatively low $(\theta=0.017)$. The experimental observation fitted perfectly the black solid line (see figure 1), this confirming what they claimed as a two-dimensional free gas (zero friction). In figure 1 it is also apparent a clear smooth transition in $\Gamma$, from a quadratic to a linear dependence on $\Delta K$, in accordance with equation (12); the motional narrowing effect is clearly observed as the friction coefficient is increased for a given value of $\Delta K$. The same behavior is also seen in figure 2, where $\Gamma$ has been plotted as a function of the shape parameter. This figure also provides an important additional information: the gradual change of the shape of the Q-peak as a function of $\chi$. Very often, in order to extract information about the diffusion mechanism, a Lorentzian shape is assumed to deconvolute the experimental results. As clearly seen in figure2, the pure Lorentzian shape is supposed to be good in a very narrow range of $\chi$ (or $\Delta K$ ) values. The three regions dividing this figure have been chosen according to the fittings of the numerical results obtained from the Langevin simulation to the analytical ones, given by equation (11). Though the two 
perpendicular border lines denoting the different shapes are somewhat arbitrary, $\chi$ values greater than one will give rise to a very strong Gaussian behavior. Therefore, this kind of representation has the advantage that experimentalists can use it as a guide in the deconvolution procedure usually carried out within this context.

The smallest experimental accessed value of the parallel momentum transfer, $\Delta K=0.21 \AA^{-1}$, corresponds to a distance of about $30 \AA$, which should be regarded as an upper limit to the mean free path of Xe atoms (or free gas regime) after reference [4]. Moreover, the experimental data for this system indicated that the Xe atoms run freely along the surface, with upper limits of $0.25 \mathrm{ps}^{-1}$ for the friction and $9 \mathrm{meV}$ for the diffusion barrier (at least less than the thermal energy). According to our theoretical study, the estimation of the mean free path should be carried out in a different way. The shape parameter and the friction can be easily obtained from a proper fit of the Q-peak to equation (11), and then the mean free path is finally computed using equation (10). For example, for $\Delta K=0.21 \AA^{-1}$ and $\gamma=0.25 \mathrm{ps}^{-1}$, one obtains that $\bar{l}=3.2 \AA$ and that the corresponding lineshape of the Q-peak displays a mixture of Lorentzian and Gaussian shapes.

The motional narrowing effect is also clearly seen in figures 3 and 4 , where numerical quasi-elastic lineshapes corresponding to different values of $\chi($ or $\Delta K$ ) are shown. Dashed lines are fittings to the numerical lineshapes according to equation (11). In particular, in figure 3, the thick black curve corresponds to a zero friction case, for which the numerical (and analytical) lineshape is a pure Gaussian function. The blue curve is obtained from a Langevin calculation with a friction $\gamma=0.25 \mathrm{ps}^{-1}$, and the best fitting to equation (11) is reached with $n_{\max }=58$, approaching a Gaussian shape. Conversely, the red curve displays a perfect Lorentzian behavior $(n=0$, with a very slight correction of the lowest part of the Lorentzian that comes from $n=1$ ). Analogously, in figure 4 , the numerical blue and red curves fit to a perfect Lorentzian $(n=0$, with an also slight correction arising from $n=1)$ and a mixed Lorentian-Gaussian function $\left(n_{\max }=10\right)$, respectively.

\section{Conclusions}

As seen in this work, the results obtained from the numerical Langevin simulations carried out by Ellis et al. [4] fit perfectly to the analytical formalism based on the Gaussian approximation of the intermediate scattering function. This allows to study the lineshape of the Q-peak in a low coverage 


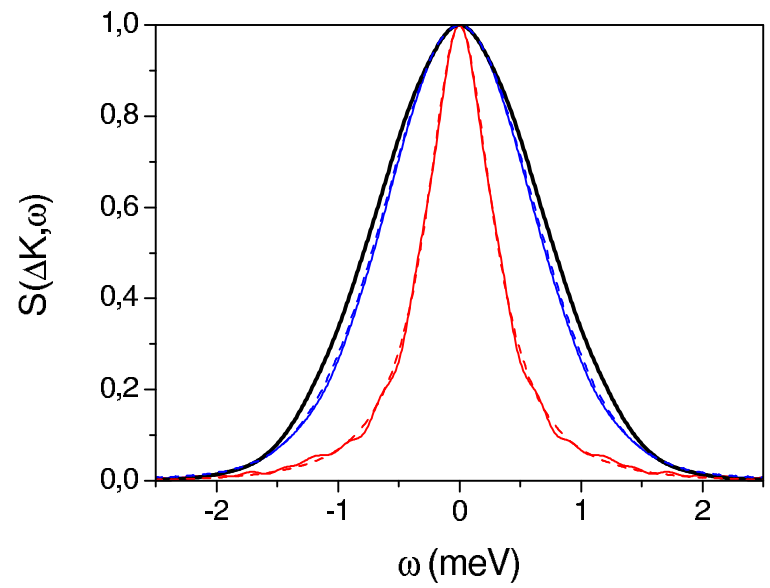

Figure 3: Dynamic structure factor, $S(\Delta \mathbf{K}, \omega)$, as a function of $\omega$ for $\Delta K=1.25 \AA^{-1}$ and three different values of the friction coefficient: $\gamma=0$ (black/thick black line), $\gamma=0.25 \mathrm{ps}^{-1}(\chi=4.1)$ (blue/thin black line), and $\gamma=2.0 \mathrm{ps}^{-1}(\chi=0.5)$ (red/thin grey line). Dashed lines are fittings to the numerical lineshapes according to equation (11).

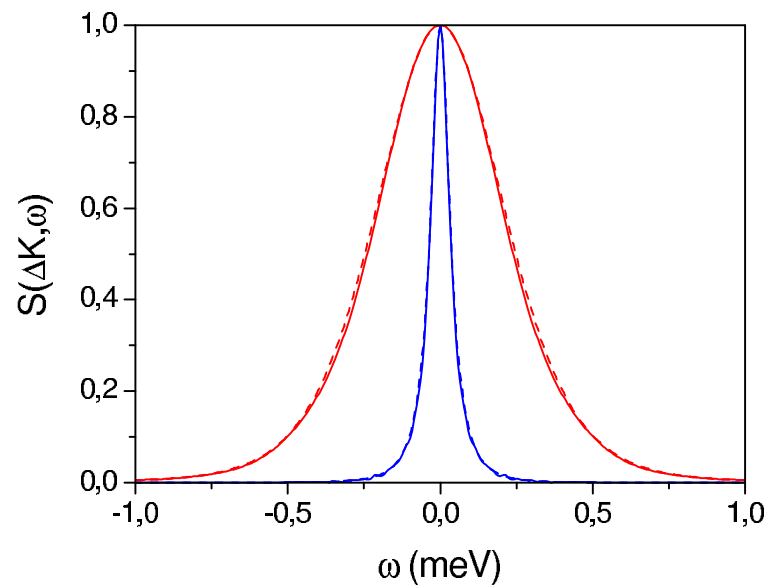

Figure 4: Dynamic structure factor, $S(\Delta \mathbf{K}, \omega)$, as a function of $\omega$ for $\gamma=0.25 \mathrm{ps}^{-1}$ and two different values of $\Delta K: \Delta K=0.15 \AA^{-1}(\chi=0.5)$ (blue/thin black line) and $\Delta K=0.5 \AA^{-1}(\chi=1.6)$ (red/thin grey line). Dashed lines are fittings to the numerical lineshapes according to equation (11). 
regime and for very low corrugated surfaces, as it is the case for the $\mathrm{Pt}(111)$ surface. Accordingly, we have shown that a gradual change of the shape of the Q-peak is expected when increasing the friction and $\Delta K$, which can be understood in the light of the so-called motional narrowing effect. This type of analysis of the Q-peak should be therefore prior to any deconvolution procedure carried out in order to better extract information from the process (e.g. diffusion coefficients and jump distributions). Moreover, taking into account these numerical simulations, conditions for the corresponding free gas regime quite different to those reported earlier have been found.

\section{Acknowledgments}

This work was supported in part by DGCYT (Spain), Project No. FIS200402461. RM-C would like to thank the University of Bochum for support from the Deutsche Forschungsgemeinschaft, SFB 558, for a predoctoral contract. JLV and AS Sanz would like to thank the Ministry of Education and Science (Spain) for a predoctoral grant and a "Juan de la Cierva" Contract, respectively.

\section{Appendix}

In this appendix we analyze the two extreme cases of the dynamic structure factor lineshape (Lorentzian and Gaussian) as a function of the shape parameter, starting from equation (11).

For $\chi \ll 1$, the dominant term in the second equality of equation (11) is that corresponding to $n=0$. Therefore, one obtains straightforwardly that

$$
S(\Delta \mathbf{K}, \omega) \approx \frac{1}{\gamma \chi^{2}} \frac{1}{1+\left(\omega / \gamma \chi^{2}\right)^{2}},
$$

which is a Lorentzian function with FWHM $\Gamma=2 \gamma \chi^{2}=2 D \Delta K^{2}$ (i.e. $\mu=1$ in equation (12)).

On the other hand, for large $\chi$, it is convenient to start with the first equality of equation (11), which is written in terms of the incomplete Gamma and Gamma functions [13, whose asymptotic behaviors are

$$
\begin{aligned}
\tilde{\Gamma}(\alpha, \beta) & \approx \beta^{\alpha-1} e^{-\beta} \\
\tilde{\Gamma}(\alpha) & \approx \sqrt{2 \pi} \alpha^{\alpha-1 / 2} e^{-\alpha}
\end{aligned}
$$


respectively, with $\alpha=\chi^{2}+i \omega / \gamma$ and $\beta=\chi^{2}$. For the sake of simplicity, it is better to express $\alpha$ is its polar form, $\alpha=\rho e^{i \delta}$, with

$$
\rho=\sqrt{\chi^{4}+\left(\frac{\omega}{\gamma}\right)^{2}}, \quad \delta=(\tan )^{-1}\left(\frac{\omega / \gamma}{\chi^{2}}\right) \approx \frac{\omega}{\gamma \chi^{2}}
$$

(the approximation in $\delta$ arises after assuming large $\chi$ ). In doing so, equations (14) and (15) become

$$
\tilde{\Gamma}\left(\chi^{2}+i \omega / \gamma, \chi^{2}\right) \approx \chi^{2\left(\chi^{2}+i \omega / \gamma\right)} e^{-\chi^{2}} \chi^{-2}
$$

and

$$
\begin{array}{r}
\tilde{\Gamma}\left(\chi^{2}+i \omega / \gamma\right)=\sqrt{2 \pi}\left[\left[\chi^{4}+\left(\frac{\omega}{\gamma}\right)^{2}\right]^{1 / 2}\right]^{\chi^{2}} \\
\times e^{-\omega^{2} / \gamma^{2} \chi^{2}} e^{i\left\{\omega / \gamma+(\omega / 2 \gamma) \ln \left[\chi^{4}+(\omega / \gamma)^{2}\right]\right\}} \\
\times\left[\chi^{4}+\left(\frac{\omega}{\gamma}\right)^{2}\right]^{-1 / 4} e^{-i \omega / 2 \gamma \chi^{2}} e^{-\left(\chi^{2}+i \omega / \gamma\right)},
\end{array}
$$

respectively. After some straightforward algebraic manipulations, equation (18) can be rewritten as

$$
\tilde{\Gamma}\left(\chi^{2}+i \omega / \gamma\right) \approx \sqrt{2 \pi} \chi^{2\left(\chi^{2}+i \omega / \gamma\right)} e^{-\chi^{2}} \chi^{-1} e^{-\omega^{2} / 2 \gamma^{2} \chi^{2}} .
$$

Substituting (17) and (19) in equation (11) we finally obtain

$$
S(\Delta \mathbf{K}, \omega) \approx \frac{1}{\gamma}\left[\sqrt{2 \pi} \chi^{-1} e^{-\omega^{2} / 2 \gamma^{2} \chi^{2}}-\chi^{-2}\right]
$$

which in the limit of large $\chi$ reads as

$$
S(\Delta \mathbf{K}, \omega) \approx \frac{\sqrt{2 \pi}}{\gamma \chi} e^{-\omega^{2} / 2 \gamma^{2} \chi^{2}} .
$$

This is a Gaussian function with $\Gamma=2 \sqrt{2 \ln 2} \gamma \chi \propto \Delta K$ (i.e. $n \rightarrow \infty$ in equation (11) and $\mu=0$ in equation (12)).

\section{References}

[1] van Hove L 1954 Phys. Rev. 95249 
[2] Vineyard G H 1958 Phys. Rev. 110999

[3] Hofmann F and Toennies J P 1996 Chem. Rev. 96 1307; Graham A P, Hofmann F, Toennies J P, Chen L Y and Ying S C 1997 Phys. Rev. B 5610567

[4] Ellis J, Graham A P and Toennies J P 1999 Phys. Rev. Lett. 825072

[5] Jardine A P, Ellis J and Allison W 2002 J. Phys.: Condens. Matter 14 6173; Jardine A P, Dworski S, Fouquet P, Alexandrowicz G, Riley D J, Lee G Y H, Ellis J and Allison W 2004 Science 104 1790; Fouquet P, Jardine A P, Dworski S, Alexandrowicz G, Allison W and Ellis J 2005 Rev. Sci. Inst. 76053109

[6] Vega J L, Guantes R and Miret-Artés S 2002 J. Phys.: Condens. Matter 14 6193; Vega J L, Guantes R and Miret-Artés S 2004 J. Phys.: Condens. Matter 16 S2879

[7] Miret-Artés S and Pollak E 2005 J. Phys.: Condens. Matter 17 S4133

[8] van Vleck J H 1948 Phys. Rev. 741168

[9] Kubo R, Toda T and Hashitsume N 1991 Statistical Physics (Berlin: Springer) Vol 2

[10] Graham A P, Silvestri W and Toennies J P 1997 in Surface Diffusion: Atomistic and Collective Processes, Vol B360 of NATO Advanced Study Institute, Ed. M Tringides (New York: Plenum) p 565

[11] Gardiner C W 1983 Handbook of Stochastic Methods (Berlin: SpringerVerlag)

[12] McQuarrie D A 1973 Statistical Mechanics (New York: Harper and Row Publishers)

[13] Gradshteyn I S and Ryzhik I M 1980 Table of Integrals, Series, and Products (New York: Academic Press) 